\title{
Meeting report Coming of age of personalized medicine: challenges ahead
} Pascal Borry

Address: Centre for Biomedical Ethics and Law, Katholieke Universiteit Leuven, Kapucijnenvoer 35, 3000 Leuven, Belgium. Email: Pascal.Borry@med.kuleuven.be

Abstract

A report on the 5th International DNA Sampling Conference 'The age of personal genomics', Banff, Canada, 16-18 September 2009.

\section{Introduction}

This 3-day conference brought together scholars involved in the ethical, legal and social controversies around the rapidly developing field of personalized genomics. More than 100 leading international experts in law, ethics, communications studies, genomic medicine and research came together for in-depth discussion on key issues, such as research ethics and governance challenges, the integration of personalized genomics into healthcare, media and popular representation of personal genomics, commercialization of personalized genomics and the regulation of direct-to-consumer (DTC) genetic testing. Here, I focus on a few topics that were discussed during the conference and that will continue to raise debate in the field of personalized genomics.

\section{Genomics and personalized medicine}

Wylie Burke (University of Washington, Seattle, USA) made the distinction between 'genomics and personalized medicine' and 'personalized genomics'. She wanted to stress that personalized healthcare is mostly not about genetics and that the main question is whether and how we can extend the paradigm of personalized medicine to genomics. She said: "Tailoring care to the individual patient is a central goal of medical practice. As new knowledge and technology develop, clinicians and policy makers have the task of considering how and when they can be used to improve the standard of care. Personal genomic testing offers several potential benefits, including new strategies for risk assessment, improved disease classification, and guidance to improve drug safety and efficacy. But such testing poses potential harms as well, and is often not the most effective strategy for personalizing care. A rigorous approach is needed for evaluating the contributions of genomics to personalized medicine."

Research ethics and personalized genomics Various speakers addressed ethical and legal standards of research. According to Donald Chalmers (University of
Tasmania, Hobart, Australia), "the success of personalized medicine and the supporting research in the Genome Era will depend, in part, on access to personal genetic information and tissue samples from a large number of patients and research participants." He asserted that "public trust and confidence in personalized medicine will depend on the maintenance of high ethical and legal standards", but also questioned whether "yesterday's research ethics standards fit the development of personalized medicine in the Genome Era." "In particular, do research ethics committees need revised guidelines on consent, weighing private and public interests, privacy, data-sharing and multiresearch or do they need greater formal regulation?" Jeantine Lunshof (Maastricht University, Maastricht, The Netherlands) advanced that "ethics and governance have built an increasingly stable compact over the past decades. Ethical, legal and social issues have gained robustness, however, at the cost of loss of flexibility and ability to adapt to the very fast progress in science." She argued that a "topdown organized steady state of biomedical ethics will not be the most adequate model for keeping pace with science."

\section{Anonymity}

Pilar Ossorio (University of Wisconsin, Madison, USA) pointed out that anonymity has always been used in research as a tool to minimize information harm: the degree of research regulation turns on whether or not specimens or data can be anonymized. "Anonymity is a policy tool that research ethics and regulation have used to balance privacy-related interests of participants with participants' and society's interests in advancing science." However, she stressed that more and more research projects will be generating whole-genome genotype and DNA sequence information linked to detailed medical information about individuals. Because whole-genome and large-scale genetic information is unique to each person and because more and more databases can be linked, anonymity cannot just be realized by stripping extrinsic identifiers. She asked the question: "If we cannot anonymize genomic data, then what policies, institutions and practices can we use to ensure that the data are broadly useful while also providing adequate protection of research participants?" She highlighted that even a very

DTC, direct-to-consumer. 
straightforward informed consent procedure does not solve this problem. Ossorio referred to the importance of adequate data-sharing policies, but also acknowledged that enforcement strategies to realize or control their implementation are weak.

\section{Developing countries and personal genomics} Abdallah S Daar (McLaughlin-Rotman Centre for Global Health, University Health Network, University of Toronto, Canada) showed, through several case studies, how emerging economies in the developing world are investing in large-scale human genomic variation studies. "Such initiatives can strengthen local research infrastructure and local intellectual property regimes, address local health needs and reduce healthcare costs, thus improving local health equity. There are, however, significant challenges to be addressed before the adoption of genomic medicine. These challenges are: the current lack of skilled human resources; ensuring sustainable funding and political will; sourcing alternative funding; improving collaboration within the public research sector as well as between the public research sector and the private sector; developing opportunities for south-south and north-south collaboration; improving the commercialization infrastructure in both the public and private sector; developing and improving the existing regulatory infrastructure; developing a healthcare infrastructure that can address access and delivery issues of genomic medicine; training healthcare workers; and engaging with the public to improve awareness and participation." Issues that rapidly need to be addressed internationally include data and sample sharing, research capacity building in developing countries, and rules and guidelines for building and using international repositories containing long-term treatment outcomes in both developed and developing nations.

\section{Direct-to-consumer genetic testing}

Various presentations focused on the issue of DTC genetic testing. Stuart Hogarth (King's College, London, UK) challenged what he termed some of the myths about the regulation of DTC genetics, such as the common conflation of regulation and proscription, the assumption that the internet has created a global market that cannot be controlled, and the idea that it is too early to intervene in this emerging market. Exploring the possibilities for regulatory intervention, Hogarth stressed the need for a minimum set of standards that should apply to all tests. Medical device regulations can ensure truthful promotion, and codes of practice can address aspects of DTC services not covered by other regulatory mechanisms. Caroline Wright (PHG Foundation, Cambridge, UK) said that "despite the increasingly crucial role of genetic tests in clinical medicine, and their growing availability directly to the public, no formal evaluation or regulatory system exists to ensure the clinical effectiveness and utility of individual tests." She underlined the need to establish a formal evaluation framework for all clinical biomarker tests, including genetic tests. Regulation of this process could take various forms: statutory legislation, codes of practice, formal guidelines or professional governance. However, the level of evidence required for tests to be allowed onto the market, to be purchased by individuals, may be lower than that required by health systems funded by the state or third parties.

\section{Conclusions}

With personalized medicine, it is hoped that the application of genomic and molecular data will help to target the delivery of healthcare, will facilitate the discovery and clinical testing of new products and will help determine a patient's predisposition to a particular disease or condition. Although personalized genomics holds great promise, it is clear that its era is only just beginning. Further understanding, elaboration and development of the ethical, legal and social issues with regard to personalized genomics are crucial in order to avoid inappropriate research and immature translation of its discoveries into (clinical) practice.

\section{Competing interests}

The author declares that he has no competing interests and was not a member of the organizing committee of this meeting.

\section{Acknowledgements}

The author is funded by the Research Fund Flanders.

Published: 24 November 2009

doi:10.1186/gm109

(c) 2009 BioMed Central Ltd 